\title{
An investigation of the low- $\Delta V$ near-Earth asteroids (341843) 2008 EV5 and (52381) 1993 HA
}

\section{Two suitable targets for the ARM and MarcoPolo-M5 space missions ${ }^{\star}$}

\author{
D. Perna ${ }^{1}$, M. Popescu ${ }^{1,2}$, F. Monteiro ${ }^{3}$, C. Lantz ${ }^{1}$, D. Lazzaro ${ }^{3}$, and F. Merlin ${ }^{1}$ \\ ${ }^{1}$ LESIA - Observatoire de Paris, PSL Research University, CNRS, Sorbonne Universités, UPMC Univ. Paris 06, Univ. Paris Diderot, \\ Sorbonne Paris Cité, 5 place Jules Janssen, 92195 Meudon, France \\ e-mail: davide.perna@obspm.fr \\ 2 Astronomical Institute of the Romanian Academy, 5 Cuţitul de Argint, 040557 Bucharest, Romania \\ 3 Observatório Nacional, R. Gal. José Cristino 77, 20921-400 Rio de Janeiro, Brazil
}

Received 10 June 2016 / Accepted 12 September 2016

\begin{abstract}
Context. The Asteroid Redirect Mission (ARM) under development by NASA is being planned to collect a multi-meter boulder from a near-Earth asteroid (NEA), and to bring it to the cis-lunar space in the mid-2020's for future study and exploitation by a crewed mission. The MarcoPolo-M5 project is being proposed in 2016 for the M5 mission opportunity by ESA, to bring back to Earth a sample from a very primitive D-type NEA. As D-types are very rare within the NEA population, considerable effort is still in progress to characterize easily accessible targets with unknown surface composition, in order to discover further asteroids that belong to this taxonomic group.

Aims. We aim to further characterize the physical properties of two optimal targets for sample return space missions, the low- $\Delta V$ NEAs (341843) 2008 EV5 and (52381) 1993 HA. The asteroid 2008 EV5 is the baseline target of ARM, but only one spectrum of this object exists in the literature. The asteroid 1993 HA is a very favourable target for a space mission based on its dynamical properties: here we intend to assess if it is a suitable target for MarcoPolo-M5.

Methods. We obtained visible spectroscopy of 2008 EV5 with the FORS2 instrument at ESO-VLT (Paranal, Chile), at different rotational phases. We also obtained visible and near-infrared spectroscopy of $1993 \mathrm{HA}$, using the EFOSC2 and SOfI instruments at ESO-NTT (La Silla, Chile). Visible photometry of 1993 HA was carried out within the IMPACTON project at the Observatório Astronômico do Sertão de Itaparica (Itacuruba, Brazil).

Results. Our new observations are in agreement with the C-type classification of 2008 EV5, which is a requirement for the ARM mission. We obtained five visible spectra which do not show any variability within the limits of noise, suggesting a homogeneous surface. We obtained the first ever spectroscopic dataset $(\sim 0.4-1.6 \mu \mathrm{m})$ for $1993 \mathrm{HA}$, finding a featureless, red-sloped behaviour typical of D-types (a T or X classification is also possible, with decreasing confidence). We also found that the synodic rotation period of $1993 \mathrm{HA}$ is $4.107 \pm 0.002 \mathrm{~h}$, a value that is optimal for the execution of a sample return mission. The derived lightcurve also suggests an elongated shape (axis ratio $a / b \geq 1.71$ ). At this stage 1993 HA does indeed seem to be the most favourable target for MarcoPolo-M5, though future observations are necessary to study it further.
\end{abstract}

Key words. minor planets, asteroids: individual: (341843) 2008 EV5 - minor planets, asteroids: individual: (52381) 1993 HA techniques: spectroscopic - techniques: photometric

\section{Introduction}

Over recent years, many scientific and technological goals have pushed space agencies to launch - and plan for the near future - space missions to near-Earth asteroids (NEAs; see, e.g. Barucci et al. 2011). However, due to the wide variety of orbital and physical characteristics of NEAs, target selection must be able to guarantee both technical feasibility and high scientific return (e.g., Perozzi et al. 2001; Binzel et al. 2004a; Ieva et al. 2014). The accessibility of potential targets of space missions from Earth is studied by classical orbital transfer algorithms: for example, the $\Delta V$ parameter (i.e. the necessary velocity change

\footnotetext{
* Based on observations collected at the European Organisation for Astronomical Research in the Southern Hemisphere under ESO programmes 290.C-5013 and 095.C-0087, and at the Observatório Astronômico do Sertão de Itaparica of the Observatório Nacional, Brazil.
}

applied to a spacecraft to realise a rendez-vous mission, following the approach by Shoemaker \& Helin 1978) can be used to identify the "easiest" targets to reach. From a scientific point of view, the primitive, carbonaceous asteroids appear to be the most appealing targets: they contain very pristine material formed in the outer solar system which can provide fundamental information on the origin and early evolution of the solar system itself (e.g. Barucci et al. 2012), and they are likely to represent a resource of water and rare minerals to be exploited by humanity in the near future (e.g. Sanchez \& McInnes 2013). Given the low number of known objects with such characteristics, the search for further low- $\Delta V$ NEAs of primitive nature has become a major topic in planetary science.

The Asteroid Redirect Mission (ARM; Abell et al. 2016) is being developed by NASA to visit an NEA with a robotic mission (to be launched in December 2021 on the current schedule), 
Table 1. Observational circumstances for spectroscopic data.

\begin{tabular}{|c|c|c|c|c|c|c|c|}
\hline Object & Telescope-Instrument & Date & $\mathrm{UT}_{\text {start }}$ & $t_{\exp }(\mathrm{s})$ & Airmass & Solar analog (airmass) & Rotational phase \\
\hline \multirow[t]{5}{*}{2008 EV5 } & VLT-FORS2 & 13 Mar. 2013 & $9: 29$ & 1440 & $2.03-1.74$ & SA 102-1081 (2.44) & 0 \\
\hline & & 15 Mar. 2013 & $9: 25$ & 1440 & $2.12-1.81$ & SA $112-1333$ (2.13) & 0.87 \\
\hline & & 16 Mar. 2013 & $9: 18$ & 1600 & $2.25-1.87$ & SA $112-1333(2.10)$ & 0.28 \\
\hline & & 17 Mar. 2013 & $9: 15$ & 1800 & $2.33-1.88$ & SA $102-1081(2.18)$ & 0.72 \\
\hline & & 22 Mar. 2013 & 9:43 & 1000 & $1.96-1.77$ & SA $112-1333(1.68)$ & 0.03 \\
\hline \multirow[t]{3}{*}{$1993 \mathrm{HA}$} & NTT-EFOSC2 & 5 Nov. 2015 & $6: 31$ & $2 \times 450$ & 1.06 & HD $11123(1.02)$ & 0 \\
\hline & & 15 Dec. 2015 & 6:01 & 600 & 1.03 & SA $98-978(1.14)$ & $0.63 \pm 0.11$ \\
\hline & NTT-SOfI & 15 Dec. 2015 & $7: 00$ & $14 \times 120$ & $1.10-1.16$ & HIP 27185 (1.12) & $0.87 \pm 0.11$ \\
\hline
\end{tabular}

collect a multi-ton boulder from its surface, and redirect it into a stable orbit around the Moon. A second crewed mission (to be launched in December 2026) will explore the boulder and return to Earth with samples. The ARM mission intends to test a number of capabilities needed for future human missions, as well as the gravity tractor technique (Lu \& Love 2005) to deflect the target asteroid orbit. The baseline target of ARM is NEA (341843) 2008 EV5, chosen because of its carbonaceous (C-type) nature and short $(\sim 4.5 \mathrm{yr})$ round-trip mission scenario. Indeed, the only available spectrum of $2008 \mathrm{EV} 5$ is typical of primitive C-type asteroids and shows a weak $(\sim 3 \%)$ spectral absorption at $0.48 \mu \mathrm{m}$, suggesting the presence of alteration minerals with similarities to the CI primitive meteorite Orgueil (Reddy et al. 2012). The albedo of 2008 EV5 (0.12 \pm 0.04 , Busch et al. 2011) is also consistent with a C-type taxonomic classification.

A primitive asteroid sample return mission has been long studied at ESA in the framework of the Cosmic Vision Programme. Hayabusa 2 (launched in December 2014 by JAXA) and OSIRIS-REx (launched in September 2016 by NASA) will return samples of a $\mathrm{C}$ - and a B-type asteroid, respectively, to Earth. Following these missions, the new proposal MarcoPoloM5 (proposed in 2016 for the M5 mission opportunity of ESA's Cosmic Vision Programme) will study a very primitive, organicrich D-type NEA (supposed to have originated in the outer solar system), and return bulk samples to Earth for laboratory analyses. This investigation will allow us to better understand the origin of planetary materials and to identify and characterize the organics and volatiles in a primitive asteroid. This will enable us to check the exobiological scenario for the origin of life which invokes an exogeneous delivery to the early Earth of complex organic molecules by primitive bodies, capable of triggering the pre-biotic synthesis of biochemical compounds on our planet. As with ARM, the target selection procedure for MarcoPoloM5 is fundamental in the mission planning, and involves both the dynamical and physical study of all the favourable targets (we note that D-type objects are very rare in the NEA population; see, e.g. Binzel et al. 2004b; and Perna et al. 2016). The baseline target for the MarcoPolo-2D proposal (not selected in 2015 for the Cosmic Vision M4 opportunity because of the M4 budgetary restrictions) was identified in the D-type 2001 SG286 (e.g., Popescu et al. 2011).

In this work we present new spectral observations of 2008 EV5, to confirm that it is indeed a good candidate target for the NASA ARM mission, as well as the first ever spectral data of (52381) 1993 HA: this asteroid offers a very efficient operational and technical mission profile, with a complete mission scenario of only $3.6 \mathrm{yr}$ (instead of the five or six years required for 2001 SG286) and could be an optimal target for MarcoPolo-M5. Further photometric observations of $1993 \mathrm{HA}$ allowed us to retrieve its rotation period and constraints on its shape. It is of utmost importance that the rotation period of the target chosen by a sample return mission should not be shorter than $\sim 2 \mathrm{~h}$, so as to be confident that a regolith is present on the surface and to have realistic expectations concerning the outcome of sampling operations. Furthermore, the rotation period needs to be fast enough to facilitate global mapping (e.g., Barucci et al. 2009).

\section{Observations and data reduction}

Visible spectroscopy of 2008 EV5 was obtained at the ESO Very Large Telescope (Paranal, Chile) using the FORS2 (Appenzeller et al. 1998) instrument and the 150I grism. Spectroscopic observations of 1993 HA were carried out at the ESO New Technology Telescope (La Silla, Chile) using the EFOSC2 instrument (Buzzoni et al. 1984) for the visible range (Grism 1) and the SOfI instrument (Moorwood et al. 1998) for the near-infrared (NIR) range (blue grism). The observational circumstances are given in Table 1. All the observations have been performed orienting the slit $\left(2^{\prime \prime}\right)$ along the parallactic angle, to minimize the effects of atmospheric differential refraction. The nodding technique of moving the object along the slit between two different positions was used for NIR observations, as is necessary at these wavelengths for a proper background subtraction.

Data were reduced using standard procedures (bias and background subtraction, flat field correction, one-dimensional spectra extraction, atmospheric extinction correction - see Perna et al. 2015, for more details) with MIDAS and IRAF packages. Wavelength calibration was obtained using emission lines from the lamps available at each instrument and which cover the spectral intervals considered. The reflectivity of our targets was then obtained by dividing their visible and NIR spectra by those of solar analogs observed close in time and in airmass to the scientific frames (cf. Table 1).

Photometric data of asteroid 1993 HA were acquired within the IMPACTON project (Lazzaro 2010) at the Observatório Astronômico do Sertão de Itaparica (OASI, Itacuruba, Brazil) on two nights, the 7 and 9 December 2015. The observations, in the R band, were made using a $2048 \times 2048$ Apogee Alta U42 CCD camera, giving a $11.8 \times 11.8$ arcmin field. The asteroid was observed for nearly $7.5 \mathrm{~h}$ using an exposure time of $120 \mathrm{~s}$ and starting at about 23:20 UT in each one of the two observing nights. During this time the airmass of the target decreased from $\sim 2$ to $\sim 1.07$, then increased again up to $\sim 1.7$ at the beginning of the morning twilight, when we stopped our observations. At the time of the observations the asteroid distance to Sun and Earth was 1.095 AU and 0.176 AU, respectively, and the solar phase was 47.4 degrees.

Data reduction was performed using the MaxIm DL package, following the standard procedures of flat-field correction and sky subtraction, and the observation time was corrected for light-travel time. 


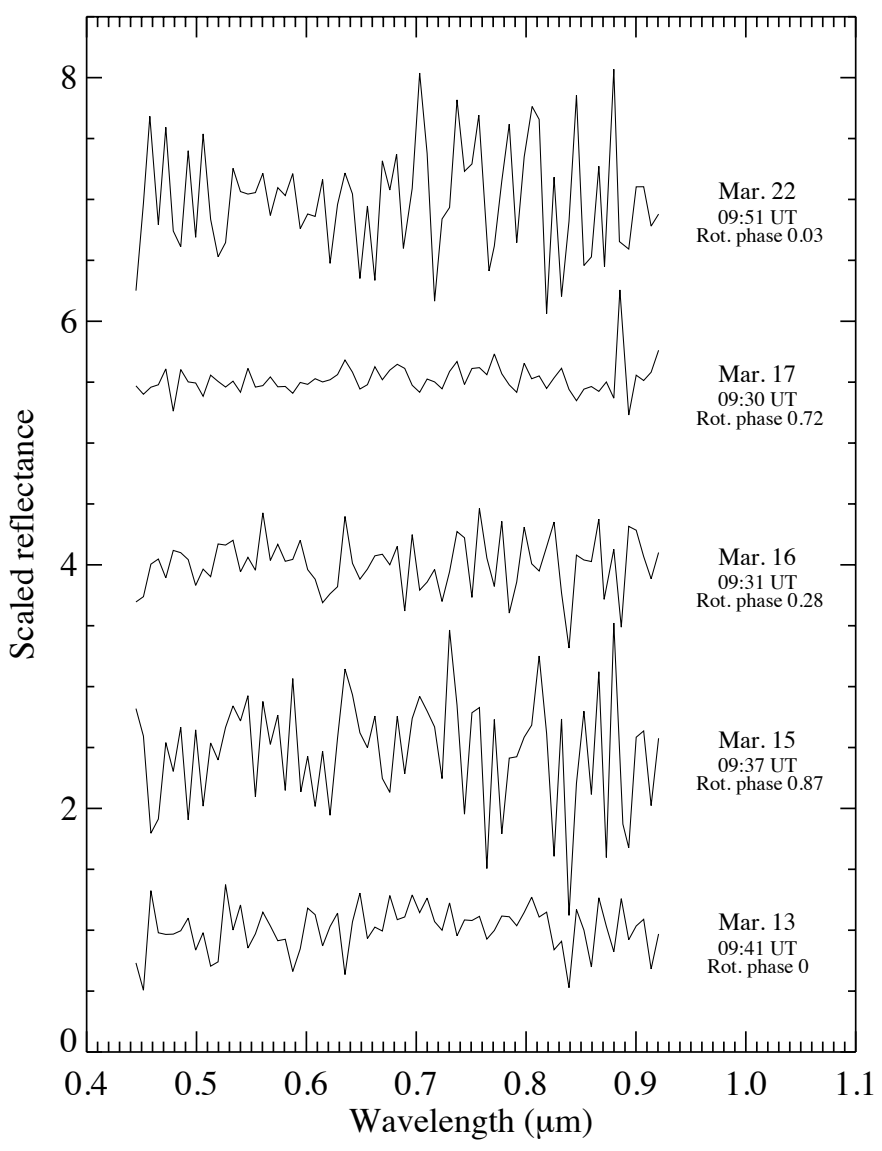

Fig. 1. Visible spectra of 2008 EV5, acquired with the FORS2 instrument at the ESO-VLT. Spectra are normalized at $0.55 \mu \mathrm{m}$ and shifted by 1.5 in reflectance for clarity. The mid-exposure time is reported for each spectrum, together with the corresponding rotational phase $\left(P_{\text {rot }}=3.725 \pm 0.001 \mathrm{~h}\right)$. We remind the reader of the low $\mathrm{S} / \mathrm{N}$ of these data (see Sect. 3.1): the spectra presented here are flat within the limits of noise.

\section{Data analysis and discussion}

\subsection{EV5}

We obtained five different spectra of 2008 EV5. The observing conditions were far from ideal, as the asteroid was observable only during the astronomical twilight, at high airmass (cf. Table 1$)$, and at a relatively faint magnitude $(V=22.2)$. Thus the resulting signal-to-noise ratio $(\mathrm{S} / \mathrm{N})$ of the reduced spectra was very low, ranging between $\lesssim 1.5$ (for the spectra acquired on 15 and 22 March 2013) and approximately three (spectrum acquired on 17 March 2013). Hence we rebinned the spectra by replacing 10-pixel intervals by their median value, in order to increase such low $\mathrm{S} / \mathrm{N}$. Figure 1 shows the final spectra, where the $\mathrm{S} / \mathrm{N}$ was improved by a factor of approximately three. The rotational phases for each spectrum are also reported, based on the rotational period of $3.725 \pm 0.001 \mathrm{~h}$ measured by Galad et al. (2009).

Despite the overall low quality of our data, they are important as the next observability window for 2008 EV5 is not until December 2020, too late for proper planning of the ARM mission. The spectra are too noisy to confirm or deny the presence of the weak $0.48 \mu \mathrm{m}$ feature observed by Reddy et al. (2012), as well as to perform a proper taxonomic classification. However these new observations of the 400-m large (Mainzer et al. 2011) 2008 EV5 suggest a flat and featureless spectrum

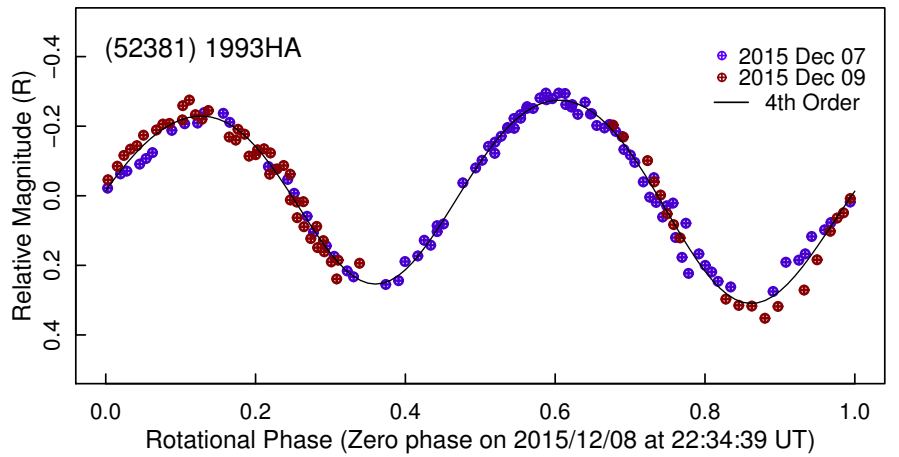

Fig. 2. Composite lightcurve of 1993 HA, folded with a synodic period of $4.107 \mathrm{~h}$. The average error on each photometric point is of $\sim 0.015$ mag.

(within the limits of noise) in agreement with the C-type classification indicated by the literature (a visible and NIR spectrum by Reddy et al. 2012; visible photometry by Somers et al. 2010 and Ye 2011). No evident variability appears with the rotational phase, providing the first evidence for $2008 \mathrm{EV} 5$ 's homogeneous surface.

\section{2. $1993 H A$}

We obtained good photometric and spectroscopic data for 1993 HA, thanks to its relatively bright magnitude at the time of our observations $(V \sim 18.2)$ and favourable sky conditions.

Two individual lightcurves were obtained over the two nights of observation via the calculation of relative magnitudes, that is, the difference between the instrumental magnitude of the asteroid and that of one field comparison star with similar magnitude. This was done in the interests of minimizing the effects of the atmospheric extinction and weather changes, resulting in a mean error of about $0.015 \mathrm{mag}$. These time-series were modelled with a 4th order Fourier polynomial (Harris et al. 1989), whose best fit relation corresponded to a synodic rotation period of $4.107 \pm 0.002 \mathrm{~h}$. The data folded with the best fit are given in Fig. 2. The double-peaked composite lightcurve is well covered with data from the two nights, and has an amplitude of $0.58 \mathrm{mag}$. From the relation $\Delta m=2.5 \log (a / b)$, where $\Delta m$ is the maximum lightcurve amplitude reached in equatorial view, we estimated a lower limit to the axis ratio of the triaxial ellipsoid shape of $a / b \geq 1.71$, implying a quite elongated body. We note that such elongation does not set any particular constraint on the execution of a sample return mission. It is worth noting that the NEA (25143) Itokawa, target of the Hayabusa sample return mission, is even more elongated (e.g., Kaasalainen et al. 2003; Demura et al. 2006).

We obtained two visible spectra $(0.4-0.92 \mu \mathrm{m})$ of $1993 \mathrm{HA}$, in November and December 2015 (Fig. 3). The latter was cut at around $0.87 \mu \mathrm{m}$ because affected by a poor sky subtraction longward. The two spectra are in very good agreement up to $\sim 0.7 \mu \mathrm{m}$, while some differences arise at longer wavelengths. The two spectra refer to almost opposite rotational phases (cf. Table 1), hence small heterogeneities on the surface cannot be excluded. The spectral slope in the $0.45-0.7 \mu \mathrm{m}$ range is $1.00 \pm 0.03 \mu \mathrm{m}^{-1}$ and $0.90 \pm 0.03 \mu \mathrm{m}^{-1}$ for the spectra acquired in November and December 2015, respectively. These values are in line with those of D-type Trojan asteroids measured by Fornasier et al. (2004, 2007). For comparison, the reddest slope measured in the same wavelength range by Neely et al. (2014) for metallic asteroids are $0.67 \mu \mathrm{m}^{-1}$ for (16) Psyche and $0.76 \mu \mathrm{m}^{-1}$ for 


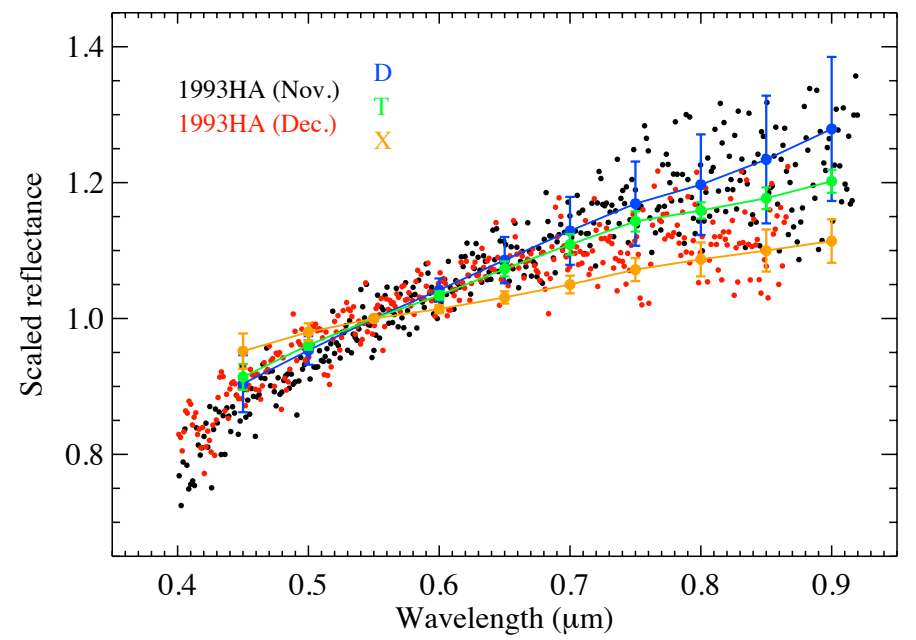

Fig. 3. Visible spectra of $1993 \mathrm{HA}$ (normalized at $0.55 \mu \mathrm{m}$ ), acquired with the EFOSC2 instrument at the ESO-NTT. The visible components of the average spectra of D-, T- and X-type asteroids from the DeMeo et al. (2009) taxonomic classification are also reported.

(441) Bathilde. This makes a classification in the X-type spectral group (whose reddest components are the metallic asteroids) unlikely, and suggests a primitive nature for 1993 HA. In Fig. 3 we also plot the visible components of the $\mathrm{D}, \mathrm{T}$ and $\mathrm{X}$ spectral types defined by DeMeo et al. (2009). We stress that such taxonomy is for objects with visible and NIR spectra, but here we use it just for visual comparison, also relying on the fact that the three plotted taxa are basically unchanged with respect to Bus' visible wavelength taxonomy (Bus 1999; Bus \& Binzel 2002).

We note that Mueller et al. (2011) have measured the size $\left(0.337_{-0.078}^{+0.097} \mathrm{~m}\right)$ and albedo $\left(0.140_{-0.077}^{+0.110}\right)$ of $1993 \mathrm{HA}$ within the ExploreNEOs survey (Trilling et al. 2010), based on observations with NASA's Warm-Spitzer space telescope. Such moderate albedo value seems marginally compatible with a D-type classification (typical albedo $\$ 0.10$, e.g., Ryan \& Woodward 2010; Mainzer et al. 2011), while more in agreement with a T- or $\mathrm{X}$-type. However it is important to consider the large error bar, as well as the fact that Harris et al. (2011) checked the accuracy of the ExploreNEOs results against values published in the literature and found albedos to be typically consistent only within $50 \%$. Hence new measurements of the albedo of 1993 HA would be extremely desirable.

The D-type classification seems to be reinforced when we take into account the NIR spectrum we obtained $(\sim 0.92-$ $1.58 \mu \mathrm{m}$ ), which has been normalized to the visible data by assuming a linear behaviour in the $0.85-1.1 \mu \mathrm{m}$ range and scaling the NIR data accordingly. We used the M4AST online tool ${ }^{1}$ (Popescu et al. 2012), and in particular the chi-square minimization method, to classify our complete visible and NIR spectrum within the taxonomic scheme by DeMeo et al. (2009). The featureless, red-sloped overall spectral shape of $1993 \mathrm{HA}$ is most indicative of a D-type asteroid $\left(\chi^{2}=0.00731\right)$, while T-type $\left(\chi^{2}=0.00840\right)$ and X-type $\left(\chi^{2}=0.01026\right)$ classes seem less compatible with the observed spectrum (Fig. 4).

\section{Conclusions}

In this work we present new observations of the low- $\Delta V$ nearEarth asteroids (341843) 2008 EV5 and (52381) 1993 HA.

\footnotetext{
1 http://m4ast.imcce. fr/
}

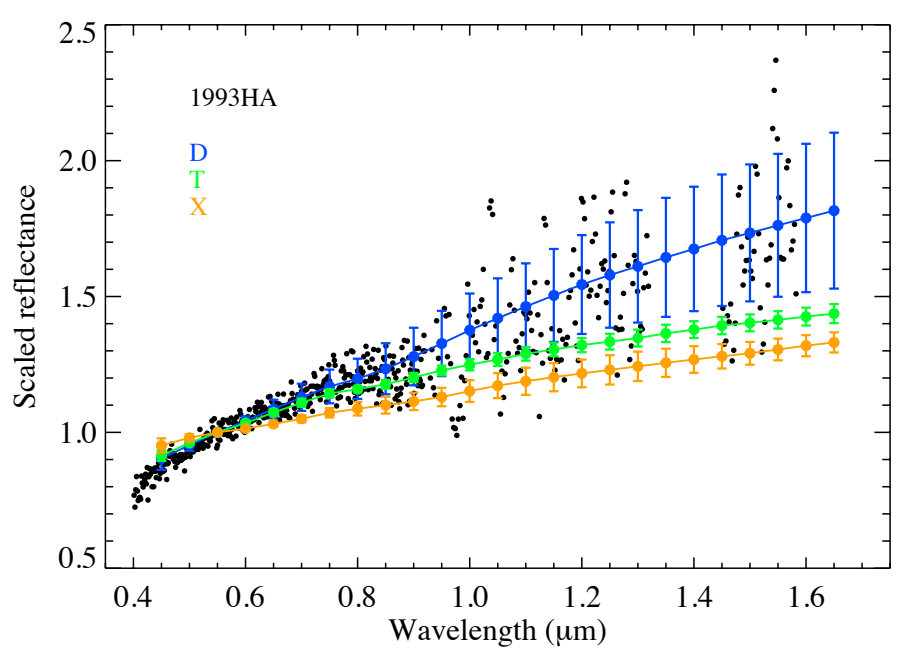

Fig. 4. Combined visible and NIR spectrum of 1993 HA (EFOSC2 + SOfI at ESO-NTT). The average spectra of D-, T- and X-type asteroids from the DeMeo et al. (2009) taxonomic classification are also reported.

The asteroid 2008 EV5 is the baseline target of the NASA Asteroid Redirect Mission project. It has been chosen mainly based on its favourable orbital properties $\left(\Delta V=5.6 \mathrm{~km} \mathrm{~s}^{-1}\right.$ following the approach by Shoemaker \& Helin 1978) and C-type spectral type. However only one spectrum of this asteroid exists in the literature, and it is important to confirm such spectral classification before investing several hundred million euros on a spacecraft mission. We note that the asteroid 2008 EV5 will not be observable again until late 2020, when the final decision about the ARM mission target will have already been made. Our new data agree with the C-type classification of 2008 EV5 and provide evidence of the homogeneity of its surface through the first rotational study of this asteroid.

The asteroid 1993 HA has never been spectrally characterized prior to our observations: our visible and NIR data suggest that this object is a rare D-type NEA. Although they are less probable, a T- or X-type classification cannot be completely ruled out, especially considering the moderate albedo value, measured roughly by Warm-Spitzer. For 1993 HA we also found a synodic rotation period of $4.107 \pm 0.002 \mathrm{~h}$, an optimal value for the execution of a sample return mission. The lightcurve amplitude of 1993 HA also implies a quite elongated shape, with a derived lower limit for the axis ratio of the triaxial ellipsoid shape of $a / b=1.71$. Considering its very low $\Delta V\left(5.3 \mathrm{~km} \mathrm{~s}^{-1}\right)$ and short mission duration (3.6 yr), $1993 \mathrm{HA}$ seems an ideal target for the MarcoPolo-M5 sample return mission proposed in 2016 in the context of the ESA M5 mission opportunity. New observations of this object (it will be as bright as $V=19.2 \mathrm{mag}$ in April 2019) will, however, be very welcome to further investigate its physical properties.

Acknowledgements. We thank the anonymous referee for the constructive remarks on the manuscript. D.P. and M.P. acknowledge financial support from the NEOShield-2 project, funded by the European Commission's Horizon 2020 program (contract No. PROTEC-2-2014-640351). F.M. thanks CAPES for its fellowship and D.L. has been supported by CNPq and FAPERJ (grants 305369/2009-1 and E-26/201.213/2014).

\section{References}

Abell, P. A., Mazanek, D. D., Reeves, D. M., et al. 2016, in Lunar and Planetary Science Conf., 47, 2217

Appenzeller, I., Fricke, K., Fürtig, W., et al. 1998, ESO Messenger, 94, 1 
Barucci, M. A., et al. 2009, Marco Polo assessment study report, http://sci.esa.int/marco-polo/46019-marco-polo-assessmentstudy-report/

Barucci, M. A., Dotto, E., \& Levasseur-Regourd, A. C. 2011, A\&ARv, 19, 48 Barucci, M. A., Cheng, A. F., Michel, P., et al. 2012, Exp. Astron., 33, 645

Binzel, R. P., Perozzi, E., Rivkin, A. S., et al. 2004a, Meteorit. Planet. Sci., 39, 351

Binzel, R. P., Rivkin, A. S., Stuart, J. S., et al. 2004b, Icarus, 170, 259

Bus, S. J. 1999, Ph.D. Thesis, Massachusetts Institute of Technology

Bus, S. J., \& Binzel, R. P. 2002, Icarus, 158, 146

Busch, M. W., Ostro, S. J., Benner, L. A. M., et al. 2011, Icarus, 212, 649

Buzzoni, B., Delabre, B., Dekker, H., et al. 1984, ESO Messenger, 38, 9

DeMeo, F. E., Binzel, R. P., Slivan, S. M., \& Bus, S. J. 2009, Icarus, 202, 160

Demura, H., Kobayashi, S., Nemoto, E., et al. 2006, Science, 312, 1347

Fornasier, S., Dotto, E., Marzari, F., et al. 2004, Icarus, 172, 221

Fornasier, S., Dotto, E., Hainaut, O., et al. 2007, Icarus, 190, 622

Galad, A., Vilagi, J., Kornos, L., \& Gajdos, S. 2009, Minor Planet Bull., 36, 116

Harris, A. W., Young, J. W., Bowell, E., et al. 1989, Icarus, 77, 171

Harris, A. W., Mommert, M., Hora, J. L., et al. 2011, AJ, 141, 75

Ieva, S., Dotto, E., Perna, D., et al. 2014, A\&A, 569, A59
Kaasalainen, M., Kwiatkowski, T., Abe, M., et al. 2003, A\&A, 405, L29 Lazzaro, D. 2010, BAAA, 53, 315

Lu, E. T., \& Love, S. G. 2005, Nature, 438, 177

Mainzer, A., Grav, T., Bauer, J., et al. 2011, ApJ, 743, 156

Moorwood, A., Cuby, J.-G., \& Lidman, C. 1998, ESO Messenger, 91, 9

Mueller, M., Delbo', M., Hora, J. L., et al. 2011, AJ, 141, 109

Neeley, J. R., Clark, B. E., Ockert-Bell, M. E., et al. 2014, Icarus, 238, 37

Perna, D., Kaňuchová, Z., Ieva, S., et al. 2015, A\&A, 575, L1

Perna, D., Dotto, E., Ieva, S., et al. 2016, AJ, 151, 11

Perozzi, E., Rossi, A., \& Valsecchi, G. B. 2001, Planet. Space Sci., 49, 3

Popescu, M., Birlan, M., Binzel, R., et al. 2011, A\&A, 535, A15

Popescu, M., Birlan, M., \& Nedelcu, D. A. 2012, A\&A, 544, A130

Reddy, V., Le Corre, L., Hicks, M., et al. 2012, Icarus, 221, 678

Ryan, E. L., \& Woodward, C. E. 2010, AJ, 140, 933

Sanchez, J.-P., \& McInnes, C. R. 2013, in Asteroids. Prospective Energy and Material Resources, ed. V. Badescu (Berlin: Springer), 439

Shoemaker, E. M., \& Helin, E. F. 1978, NASA Conf. Publ., 2053, 245

Somers, J. M., Hicks, M., Lawrence, K., et al. 2010, BAAS, 42, 1055

Trilling, D. E., Mueller, M., Hora, J. L., et al. 2010, AJ, 140, 770

Ye, Q. 2011, AJ, 141, 32 